\title{
Research on Pricing Strategies of Chinese E-Business Platforms Based on Game Theory
}

\author{
Tianyi Chang ${ }^{1, *}$, a, $\dagger$ Ying $\mathrm{Guo}^{2, *, b, \dagger}$
}

\author{
${ }^{1}$ Beijing Huijia private school Beijing 100000 China \\ ${ }^{2}$ Southwest University Chongqing 400715 China

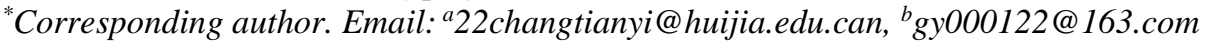 \\ ${ }^{\dagger}$ These authors contributed equally.
}

\begin{abstract}
E-Business platforms have developed rapidly in recent years, providing consumers and enterprises with a virtual trading environment, greatly facilitating people's consumption behavior and reducing transaction costs. And this flexible trading method also provides a variety of possibilities for commodity pricing. The pricing strategy of the platform has a strategic impact on both sides of consumption and the operation and profitability of the platform company itself. This article studies the popular pricing strategies in recent years, consults relevant information, and summarizes the importance of using big data analysis and optimizing existing pricing strategies. And decided to use relevant game theory knowledge to analyze the pros and cons of existing pricing strategies to arrive at an optimization plan. This article mainly uses research methods such as literature research, case study, comparison, and contrast in the research process. According to the development status of China's E-Business platform, it analyzes the advantages of existing price strategies regarding the use of discounts. It proposes that the price strategy is relatively traditional without innovation, consumer groups are unstable, and low prices easily lead to low credit and other problems. After discussing this article, we hope to solve these problems from the perspective of changing traditional methods, catering to new consumer concepts, stabilizing consumer groups, and formulating reasonable price strategies. From an academic point of view, this article proposes a new idea to study the price strategy of China's E-Business platform, and the combination of knowledge and practice of game theory has provided new reference materials for other scholars. In addition, it also helped China's E-Business platform find a better development direction. Combining big data analysis and dynamic price strategy will also help China's E-Business industry develop more stably and at high speed.
\end{abstract}

Keywords: Game Theory, E-Business platform, Pricing issues, Strategy

\section{INTRODUCTION}

To begin with, the E-Business platform is the platform that conducts any business using the internet, extranet, web, and intranet. People can see the EBusiness platform everywhere, and the prestigious examples are Taobao or Jingdong. According to current data, the sum of the active user for the top twenty EBusiness platforms is more than seven hundred and twenty-four million (it's possible for overlapping of users) [1]. Also, In 2019, China's E-Business transaction volume reached 34.81 trillion yuan, increasing $6.7 \%$ year on year [2]. By looking at those data, people should realize that the E-Business platform has already become an indispensable part of their lives. Hence, researching the pricing strategy may help people do the most beneficial or the least harmful decision while facing a trade-off decision. In the field of pricing strategy, a lot of outstanding researchers from all over the world contribute their idea.

To be more specific, Zhou and Huang proposed that the E-Business can lower the cost for the transaction between sellers and buyers, which further makes business more approachable to the consumer. More than that, they also pointed out a type of pricing strategy, which is a dynamic pricing strategy, and it allows the seller on the E-Business platform to manipulate the price of the goods based on consumer's consuming preferences, which the big data might gather. Through using the dynamic pricing strategy, the seller can obtain 
all of the consumer surpluses. For example, the one percent increase in the price of the goods can bring a 7.4\% increase in the company's profit [3]. Moreover, according to another group of experts, Gong and Liu, found that the pricing strategy of some of the EBusiness plays for convert from both of the sellers and buyers free to only one of them free because, with the development of the E-Business platform, people usually desire higher quality service. For the pricing strategy, Liu and Gong also chose to diversify the potential consumer and provide different consumer personalized prices based on their consumption habits to maximize profit. Also, they mentioned that due to the importance of knowing the consumption preference of the consumer, the E-Business platform should also start to develop its own big data technology [4]. According to another research paper by Xia, he criticized the conventional pricing strategy that the E-Business platform uses. To be more specific, he proposed that doing decisions based on experience is ineffective because uncertainty might happen. Furthermore, he also pointed out the drawback of unidirectional marketing, sending Ads to people randomly, making the consumer feel annoyed. After analyzing the disadvantages of the traditional, Xia proposed the importance of the big data analysis to the pricing strategy by quoting an American scholar, Jef Sabin, and he pointed out a term called "precise marketing," which can be understood as sale goods to a certain group consumer[5]. Based on the research that we did, we find out that there are similarities between the research conducted by different researchers. They use big data to calculate consumer preference and the dynamic pricing strategy, which can also be known as determining the personalized price for a different consumer. Those researches pointed out the development trend for the Chinese E-Business platform further, which is to develop their own big data technology. However, there is still a lack of research on the pros and cons of different pricing strategies, especially game theory. According to the researches, there are huge differences between the conventional pricing strategy and the modern pricing strategy, so to encourage those different E-Business platforms to make changes, it is necessary to provide a comprehensive game theory analysis for using the "dynamic pricing strategy" or "developing the big data technology".

In this article, we will mainly adopt three research methods: literature research, case study, and comparison and contrast, and each of them plays an extremely significant role. We will be dedicated to reporting the current developments of the Chinese E-Business platform, and based on the background of game theory to points out the potential problems for the different pricing strategies, and thus analyze the reasons that lead those problems, and at last optimize the price strategies. For the expected result, we expect after the research. We can point out the pros and cons of different pricing strategies and propose one that is proper for Chinese EBusiness development.

\section{METHODOLOGY}

When discussing the pricing strategies of E-Business platforms and analyzing their problems, this article starts from a systematic and global perspective, combined with the discussions of some scholars in the past, and analyzes the existing pricing strategies of multiple E-Business platforms. It is worth noting that this article combines literature research, case study, comparison and contrast, and other methods. And it has a certain degree of innovation in research methods than previous scholars. In addition, in the research on the price strategies of the existing E-Business platform, this article further discusses and analyzes the possibility of optimizing the pricing strategy of the E-Business platform and the specific plan, which also has a greater breakthrough in the selection of the subject.

\subsection{Literature research}

Literature research is a method of collecting, identifying, and collating literature and forming a scientific understanding of facts through the literature study [6]. This article refers to some documents discussing the price strategies of E-Business platforms.

To begin with, we read some news and industry reports. After understanding some development status and prospects, we decided to establish the research object as the E-Business platform, the most popular industry. Then, when searching for information, we found some journals, which are our main source of information for understanding the pricing strategies of existing E-Business platforms. In the literature review process, according to some of the articles mentioned, we realized that dynamic price strategies relying on big data analysis to calculate consumer preferences are the best development direction for E-Business platforms. Besides, through the study of "A Primer in Game Theory"[7], we use the knowledge of game theory to analyze the price strategy and optimization of EBusiness platforms.

Through the literature research method, we chose the research industry and specific analysis objects and learned about the existing pricing strategies of several E-Business platforms. This article conducts a more comprehensive and in-depth discussion on this basis and optimizes the price strategy of E-Business platforms from the perspective of game theory.

\subsection{Case study}

The case study conducts an in-depth analysis of a specific case in a related field to summarize the experience, discover problems, and conduct more in- 
depth thinking[8]. From a realistic point of view, this article uses "Taobao" as an example to analyze the basis of online marketing pricing and conduct specific analysis and discussion on some of its current price strategies. Using a case study to analyze representative companies in the industry makes it easier to draw the targeted and specific conclusion that what is the problem with the existing price strategy and which kind of price strategy is more suitable for the current EBusiness platform.

\subsection{Comparison and contrast}

Comparison and contrast are to compare objective things to understand the nature and laws of things and make correct evaluations[9]. We analyzed Taobao's current online marketing price strategies and compared its traditional pricing strategy to summarize the differences over the past few years. On the one hand, it is more convenient for us to understand the development direction of Taobao in recent years and summarize the mainstream development trend of the current E-Business platform price strategies. On the other hand, we can determine what problems still need to be solved and from which angle to start to optimize the price strategy.

\section{RESULTS}

\subsection{The development status of China's $e$ - commerce platform}

The current development of the E-Business platform is in the fast lane, and based on the reliable data, the sales volume for the Chinese E-Business platform grows to 7323.7 billion yuan (2019), a year-on-year increase of $16.8 \%[10]$. Moreover, the E-Business platform also becomes more and more pluralistic than before in consumer groups and products. To be more specific, the target consumer for the E-Business platform includes all ages from young to old, and their main business has penetrated into every corner of our life.

\subsection{Advantages and problems of pricing strategy of China's e-commerce platform- Taobao as an example}

In China, there are countless E-Business platforms, and each of them shares some similarities and some differences in a certain range. There are two most famous and richest E-Business platforms in China: Jingdong and Taobao, and each of them play an extremely important role in our lives. Under the big trend, either Jingdong or Taobao developed their own operating mode. In this article, we will discuss the advantages and disadvantages of the pricing strategies of the E-Business platform in China based on Taobao.

\subsubsection{Advantages}

\subsubsection{Use of discount}

In the E-Business platform, we can see that many products have discounts frequently, and in this case, the consumer will be more willing to buy the products since they want to gain the advantage. TaoBao uses a discount strategy extremely well, we can see that numerous products are discounting, and through this, the seller will be able to attract more attention from different consumers. However, based on the researches done by Paidai net, the author proposed that "even though discount will bring the decrease of the profit or even lose sales, but the popularity that it brings will benefit the sale of other products in the seller's store[11]."

Therefore, we may see that there is one thing crucial for the E-Business platform, which is "clicks". In other words, the discount of a single product will bring the increased sale of a product and bring popularity for the whole store, and in this case, the seller may earn more from selling other products rather than the product that has a discount. Therefore we can see that in the EBusiness platform, stream, which refers to a certain period to open the site address popular visits, is also a unique advantage.

\subsubsection{Cancelling the agency fee}

Because Taobao is a media that provides the third party seller an opportunity to trade with buyers, therefore, usually the middleman media will charge both sides (seller and buyer) for extra money. According to researcher Yu, E-bay has also tried to occupy the Chinese market. However, compared to Taobao, which does not ask for the transaction fee, E-bay loses the Chinese market[12]. Therefore, through analyzing this incident, we can see that one of the biggest advantages of Taobao's official pricing strategy is that they don't want to ask for intermediary fees. Yu also mentioned that in China, although e-bay has launched a series of policies to adapt to China's localized market, such as the launch of eBay[12]. However, Taobao encourages Chinese consumers to be confident about the E-Business platform by cancelling the agency fee. As a result, Taobao controls over 60 percent of the market share, much more than eBay (less than eBay 30 percent)[12]. Even though Taobao changes this policy right now, we still need to admit the huge benefits of canceling the agency fee to the infant industry.

\subsubsection{Problems}

\subsubsection{The price strategy is too conventional.}

With the development of the trade mode, more and more people have become familiar with some of the strategies that the seller uses to grab the consumers' 
attention. Thus, it keeps using the same kind of strategy to create consumers' resistance since they have faced the same strategy for a long time. To be more specific, end the commodity price as 99 , which is the pricing method adopted by Western retailers based on the study of psychology. There are mainly two major functions for this price strategy. First, it can make the consumer think that the store has a serious and accurate sales price, and even if it is close to the integer, it still ends as 99 . Secondly, it can make the consumer feel that the goods are "cheaper". For example, 9 cents is something with a few cents, and one yuan is more than one yuan of goods. The 99 number can promote the customers to have the desire to shop[13]. Based on the descriptions for the 99 pice strategy, we may find out that it works extremely well in both the online and offline shop because it manipulates people's desire for buying the product. However, we should not underestimate the resistance of the consumer. This means that since the seller adopts the same strategy for a long time, the consumer will notice the trap of the price strategy. Keep using the 99 price strategy as an example, assuming that this strategy has been used for a long time and all consumers know this trick. Hence, the consumer won't still consider the product that adapts the 99 pricing method as either accurate or cheaper.

\subsubsection{Uncertainties of consumer groups}

We all know that targeting a specific group of consumers is the key for both the E-Business platform and the offline stores because the seller needs to provide a certain kind of service for a specific class of consumers to improve efficiency. However, the EBusiness platform does not always operate following the above strategy. This is because the cost of placing goods on "shelves" is almost zero, so the E-Business will always set varied goods with different prices in their store following a ratio called 2:7:1. To be more specific, based on the book that published by Gong, he suggests that the E-Business platform seller should set their price according to the 2:7:1 theory, which separates the goods in the E-store into three categories [14]. In other words, the goods are categorized by different prices, and $20 \%$ of the products are mainly used for drainage and activities. Setting a low price is to attract traffic through relatively low prices or disturb the price system in the minds of consumers so that consumers feel that the cost performance of the whole store is relatively high. $70 \%$ of the proudest in the main force for the store, and its price is medium refers to the average level of the industry's overall market. The rearming $10 \%$ of the products are high priced, and this part of the products is brand image, but those usually are not expected to be sold. With a price strategy like 271, the E-Business stores will sell the product at hugely different prices, so they won't have a certain group of consumers. Hence, the E-Business seller will face expensive advertising or servicing costs since the consumers are all different. There are no ways for them to utilize the homogeneous work to different products.

\subsubsection{Over low price lead to the consideration of consumers.}

Price is always the more effective way to compete with others, so the sellers have used the price war thousands and thousands of times to grab consumers' attention. However, people always say that the cheap thing is usually of bad quality. Thus the over low price might lead the extra consideration of consumers because they might think the things with low price normally have low cost. To be more specific, the products in Taobao are kind of cheap because we can see that there is one in each of the items, either food or clothes are extremely cheap, and maybe the seller's aim is to the seller more with a cheaper price. Still, the consumer does not know what the seller is thinking. Therefore, the consumer will assume the product is inferior and then stop buying it.

\subsection{Analysis of the Causes of the Pricing Strategy Problems of China's E-Business Platforms}

\subsubsection{The E-Business platform is not creative for the price strategy.}

Some of the conventional price strategies have existed for a long time. It worked very well in some of the offline stores. Thus, the E-Business platform seller over-relying on the strategies that derive from the offline store. Since the consumer starts to resist the conventional price strategies, the E-Business platform seller must invent new price strategies to meet consumers' demand.

\subsubsection{E-business platform sellers dream of price diversification for their products.}

The E-Business platform sellers dream of diversifying their products to seek more profit, or some of the new infant industry want to establish good fame for their brand. As a result, they chose to set different price levels for their products.

\subsubsection{Over competition for the E-Business seller}

Since the E-Business becomes more and more popular, the number of sellers on the E-Business platform also increases, intensifying the completion there. Therefore, to become the winner in the competition, the sellers adapt to the price war, which is the potential reason for the products to be over cheap. 


\section{DISCUSSION}

In this article, we take Taobao as an example to summarize the main problems of the current price strategies of Chinese E-Business platforms, discuss and analyze them, and find out the corresponding solutions.

\subsection{Change the traditional pricing strategy to cater to the new consumption concept.}

After discussing and analyzing the development status of China's E-Business platforms, we realized that several of the more traditional price strategies currently in use are somewhat out of date. With the continuous development of the national economy, consumers have begun to have higher spending power, and consumption concepts have also changed. The increase in consumption power has resulted in price no longer being the only criterion for consumers' consumption process. Traditional price strategies still remain at the level of low prices and preferential treatment. For example, traditional price strategies that lack innovation, such as the 99 pricing method and the low price method, have been unable to attract most consumers. There are also price strategies such as spike pricing, bundled pricing, and pre-sale pricing that have been popular in the past two years. Initially, they can effectively stimulate consumers' desire to buy and help products quickly occupy market share, but there will always be a wave of refunds after every major discount event.

We can use the method of differentiated pricing of commodities to specific price concessions to each commodity to avoid impulsive consumer consumption and invalid consumption. Only implement a price strategy similar to secondary price discrimination for the same type of goods while only knowing part of each other's information, allowing consumers and businesses to have more flexible and subjective choices [15]. In this way, the efficiency of the transaction is improved, but unnecessary losses such as freight are reduced. This more flexible price strategy caters to the new consumption concept, allowing consumers to feel more respect when making transactions, and it is not easy to experience regret and disgust.

\subsection{Improve the pertinence of pricing strategies and stabilize the consumer population.}

At present, many E-Business platforms like to adopt a penetration price strategy, setting different prices in different periods. This kind of price strategy can play a role in attracting traffic, attract consumers, and occupy market share in the early stage of product launch. In addition, it also helps to establish a more influential product image in the later stage [16]. However, this highly differentiated price strategy can easily lead to instability of the consumer population, and most consumers who are more sensitive to price changes may be lost before building brand loyalty. Consumer groups in different periods are from different classes, and this unstable consumer group is not conducive to building a brand image. In this regard, we believe that differentiated pricing of products is reasonable, but differentiated services should also be provided at the same time. Stabilizing consumer groups of different classes in the early stage of brand establishment is also more conducive to establishing a reliable and friendly brand image. Continuously improve the quality of service, and achieve long-term operating benefits while bringing a good value-added experience to consumers.

\subsection{Reasonable pricing instead of blindly pursuing low prices}

In the E-Business platform, many suppliers provide homogeneous products. To win in the competition, they often adopt the method of lowering prices. Although this method can temporarily improve market competitiveness, it also reduces profits and may lose consumer trust in the brand. In the past, low-price strategies can help new products occupy a larger market share while also enhancing market competitiveness and defeating other competitors. In the new consumption concept, brand image and product quality have become more important, which requires platforms not to set prices too low when setting prices. Because consumers generally believe that product quality and price are positively correlated. Too low prices will make consumers question the goods and reduce their desire to buy. Therefore, the supplier's reasonable pricing is more conducive to cultivating consumer trust and loyalty to the brand. Therefore, for long-term development considerations, a certain increase in product prices based on cost is a win-win strategy for consumers and sellers. The degree of improvement should be determined according to the consumer's preference for products and the elasticity of demand obtained by big data analysis. The price of products with a high degree of preference or relatively small demand elasticity can have more room to increase. At the same time, the price of products with a low degree of preference or relatively large demand elasticity cannot be increased too much[17].

\section{CONCLUSION}

\subsection{Summary of main findings}

In a nutshell, the main findings of this article can be divided into two sections, which are results and discussion.

In the result section, we point out two advantages and three advantages for the price strategy of the 
Chinese E-Business platform based on the case study of Taobao. The two advantages are the use of discounts and canceling the agency fee. The former is the price strategy used by the seller on the E-Business platform to attract more consumers to purchase its products. The latter is the price strategy used by the E-Business platform to attract both sellers and buyers. For its disadvantages, we have summarized three of them. The first one is that the price strategy is too conventional, which might attract consumers. The second disadvantage is that it will lead the uncertainties of the target consumer through selling different valued products. The third and last disadvantage is that the over cheap price for the product will lead to the extra to consider from the consumers since they will wonder why it is so cheap.

In the discussions section, we made three suggestions corresponding to the three problems that we pointed out. The first solution is to change the traditional pricing strategy to cater to the new consumption concept. The second solution is to improve the pertinence of pricing strategies and stabilize the consumer population. The third solution is to have reasonable pricing instead of blindly pursuing low prices.

\subsection{Limitation}

For the limitation, the overall article is based on secondary materials, and we quote a lot of information from other researchers. Therefore, compared to the primary materials, which we prefer to investigate by ourselves, the reliability is limited. In the future, we will still be dedicated to dealing with the price strategy of the Chinese E-Business platform and further point out more problems and the corresponding optimal solutions.

\section{REFERENCES}

[1] Unknown, Top 20 list of active users of comprehensive e-commerce app released in October 2020, Souhu, 2020.

[2] Zheng Chen, Analysis on the development status of China's e-commerce market in 2020, Forward The Economist, 2020.

[3] Zhou Jin, Huang Lingping, Research on dynamic pricing strategy of E-Business enterprises, Price theory and Practice, 2008, DOI:10.19851/j.cnki.cn11-1010/f.2008.07.039.

[4] Gongfang, Liu Xiangli, Research on pricing strategy of o2o e-commerce enterprises based on big data, Market modernization, 2017, DOI : CNKI:SUN:SCXH.0.2017-16-007

[5] Xiawen, Strategy Research of business enterprise based on big data precision marketing, value engineering, 2018, OI:10.14018/j.cnki.cn131085/n.2018.32.040

[6] Definition of literature research, Baidu, https://baike.baidu.com/item/\%E6\%96\%87\%E7\%8 C\%AE\%E7\%A0\%94\%E7\%A9\%B6\%Е6\%B3\%95

[7] Gibbons, R. , A Primer in Game Theory, China Social Sciences Press, 2015

[8] Definition of case study, Baidu, https://baike.baidu.com/item/\%E6\%A1\%88\%E4\% BE\%8B\%Е5\%88\%86\%Е6\%9E\%90\%Е6\%B3\%95

[9] Definition of comparison and contrast, https://baike.baidu.com/item/\%E5\%AF\%B9\%E6\% AF\%94\%E6\%B3\%95/3730682?fromtitle $=\% \mathrm{E} 5 \% \mathrm{~A}$ F\%B9\%E6\%AF\%94\%E5\%88\%86\%E6\%9E\%90\% E6\%B3\%95\&fromid=9111964\&fr=aladdin

[10] unknown, Analysis report of China's e-commerce industry in 2021 - market depth analysis and development prospect research, Chinabaogao, 2021.

[11] my_world88, Analysis of Taobao product pricing strategy,Paidai, 2012.

[12] Yuxia, sustainable research about Taobao Platform ' s pricing strategy, appllied economics, 2012, pp. $1-2$.

[13] Liu yixin, The latest store, China Chang'an Publishing House, 2008.

[14] Gong wenxiang, How traditional enterprises do ecommerce and micro E-Business, Electronic Industry Press, 2015, pp. Chapter II section 2, ISBN 978-7-121-26682-9.

[15] Li Zhiqiang. On the pricing strategy of e-commerce [D], Foreign Economies\&Management, 2002, pp. Volume 24, issue 10.

[16] Fu xingmei, Pricing behavior and differentiated competition strategy of e-commerce platform, Research on commercial economy, 2019, pp. 7275.

[17] $\mathrm{Fu}$ xingmei, Pricing behavior and differentiated competition strategy of e-commerce platform, Research on commercial economy, 2019, pp. 7275. 